\title{
Prevalence of methicillin-resistant Staphylococcus aureus among health care workers of intensive care units in Ecuador
}

\author{
Alejandra Ruiz ${ }^{1}$, Marcelo Mora ${ }^{2}$, Camilo Zurita ${ }^{2,3}$, Danny Larco ${ }^{2}$, Yadira Toapanta ${ }^{2}$, Jeannete Zurita ${ }^{2,4,5}$ \\ ${ }^{1}$ Facultad de Ingeniería en Biotecnología, Escuela Politécnica del Ejército, Sangolquí, Ecuador. \\ ${ }^{2}$ Centro Internacional de Investigaciones en la Salud-Ecuador, Quito, Ecuador. \\ ${ }^{3}$ Facultad de Medicina Universidad Central del Ecuador, Quito, Ecuador. \\ ${ }^{4}$ Servicio de Microbiología y Tuberculosis. Hospital Vozandes, Quito, Ecuador. \\ ${ }^{5}$ Facultad de Medicina de la Pontificia Universidad Católica del Ecuador, Quito,Ecuador.
}

\begin{abstract}
Introduction: Colonization of health care workers with methicillin-resistant Staphylococcus aureus (MRSA) has been an important route of dispersion and infection of MRSA and has been implicated in epidemic outbreaks. The objective of the present study was to assess prevalence of MRSA colonization in the anterior nares of health care personnel at the intensive care unit (ICUs) of three hospital facilities in Quito, Ecuador.

Methodology: The prevalence of MRSA in specimens from all ICU health care workers of three hospitals was measured by using a real-time PCR assay and CHROMagar MRSA.

Results: The prevalence of MRSA among the three health care facilities was $2.4 \%$.

Conclusion: The prevalence of MRSA colonization was relatively low compared to other studies and showed no differences between hospital facilities.
\end{abstract}

Key words: methicillin-resistant Staphylococcus aureus (MRSA); intensive care unit; health care facility

J Infect Dev Ctries 2014; 8(1):116-119. doi:10.3855/jidc.3535

(Received 12 March 2013- Accepted 19 June 2013)

Copyright (C) 2014 Ruiz et al. This is an open-access article distributed under the Creative Commons Attribution License, which permits unrestricted use, distribution, and reproduction in any medium, provided the original work is properly cited.

\section{Introduction}

Health care workers are an important link between hospitals, nursing homes, and communities in nosocomial infections [1]. They have been traditionally considered as vectors of nosocomial pathogens. Methicillin-resistant Staphylococcus aureus (MRSA)is considered to be one of the most serious hospital infections [1] and has raised alarms in many hospital facilities because of potential epidemic outbreaks.

Intensive care units (ICUs) are among the hospital departments that have reported high levels of MRSA colonization in patients and health care workers $[2,3]$. Understanding the frequency of colonization of nosocomial pathogens in ICUs is essential because ICUs have a considerable number of critical and immune-suppressed patients. The objective of the present study was to assess the frequency of MRSA colonization in the anterior nares of ICU health care personnel in three hospital facilities in Quito, Ecuador. Anterior nares were used in this study because they have been reported as one of the sites with the highest sensitivity for detecting MRSA colonization [4].

\section{Methodology}

Specimens were taken with Dacron-tipped swabs from the anterior nares of available health care workers from the ICUs of three hospital facilities in Quito, Ecuador: hospital A, hospital B, and hospital C. The same swab was inserted into both nares $(1 \mathrm{~cm})$ and rotated five times. The swabs were immobilized within the anterior nares for 15 seconds to ensure that the interior nares were in contact with the swabs. The study was done with the authorization of the ethical committee of each hospital. All participants signed an informative consent form. Data was collected through a confidential survey and included demographic characteristics (gender and age), length of service, and employee occupation. 
Table 1. Frequency of MRSA cases detected using real-time PCR LightCycler MRSA advanced test at three hospital facilities

\begin{tabular}{llcc}
\hline Institution & Employee occupation & Number of participants (\%) & MRSA cases (Frequency) \\
\hline Hospital A & Administration staff & $4(3.7 \%)$ & \\
& Nursing staff & $60(55 \%)$ & \\
Medical staff & $22(20.2 \%)$ & \\
& Others & $7(6.4 \%)$ & $3(2.8 \%)$ \\
& Physiotherapy staff & $16(14.7 \%)$ & \\
Hospital B & Total & $109(100 \%)$ & \\
& Administration staff & $4(7 \%)$ & \\
& Nursing staff & $23(40.3 \%)$ & \\
& Medical staff & $25(43.9 \%)$ & \\
& Others & $5(8.8 \%)$ & \\
& Physiotherapy staff & $0(0 \%)$ & \\
& Total & $57(100 \%)$ & \\
Hospital C & Administration staff & $2(4.5 \%)$ & $1(2.3 \%)$ \\
& Nursing staff & $29(65.9 \%)$ & 0 \\
& Medical staff & $7(15.9 \%)$ & $3(2.7 \%)$ \\
& Others & $5(11.4 \%)$ & 0 \\
Physiotherapy staff & $1(2.3 \%)$ & $1(5.9 \%)$ \\
Total & Total & $44(100 \%)$ & $1(5.9 \%)$ \\
& Administration staff & $10(4.8 \%)$ & $5(2.4 \%)$ \\
\hline
\end{tabular}

Swab specimens were conserved in Stuart's medium (COPAN Venturi Transystem Copan, Brescia, Italy) and stored at $-20^{\circ} \mathrm{C}$ until processed. Swabs were streaked on a CHROMagar MRSA (Difco Laboratories, Detroit, USA) plate and incubated for 24 hours at $35^{\circ} \mathrm{C}$. The growth of colonies showing any pink or mauve coloration indicated the swabs were positive for MRSA. Additionally, positive colonies were isolated in mannitol salt agar (Difco Laboratories, Detroit, USA), and susceptibility tests were done to confirm methicillin resistance.

DNA extraction was done using a LightCycler Advanced Lysis Kit (Roche Diagnostics Mannheim, Germany). MRSA detection was done using real-time amplification according to the protocol LightCycler MRSA advanced test (Roche Diagnostics, Mannheim, Germany). For analysis, participants were grouped into five categories: administration staff, physiotherapy staff, nursing staff, medical staff, and others (cleaning and security staff). Statistical differences between job groups were analyzed by Kluskal-Wallis test using INFOSTAT software at a 0.05 level of significance.

\section{Results}

Two hundred and ten samples were taken from participants working at ICU at three health care facilities. Participants had an average age of $35 \pm 10$ years, and $71 \%$ of them were females. Hospital A had 109 participants, hospital B had 57 participants, and hospital $\mathrm{C}$ had 44 participants (Table 1). Five MRSA isolates were detected using the real-time PCR LightCycler MRSA advanced test. In contrast, only three MRSA isolates were detected using CHROMagar MRSA.

No significant differences were observed between job group, age, or length of service. Nursing staff (the group with highest number of participants) reported three cases in hospital A, and no cases in hospitals B and $C$ (Table 1). In spite of being the group with the second highest number of participants, medical staff did not report any MRSA cases. Physiotherapy and others reported one case each.

The three ICUs had similar MRSA frequencies (Table 1) and did not show statistical differences ( $p>$ 0.05). The frequency of MRSA among the three health care facilities was 5/210 (2.4\%) using real-time PCR methodology and 3/210 (1.4\%) using CHROMagar MRSA. 


\section{Discussion}

Even though comparisons between studies are difficult because of the big differences in numbers of participants, low rates of MRSA colonization in the ICUs of three health care facilities $(3 / 109,1 / 57,1 / 44)$ were similar to the frequencies found in other ICU studies in Italy $(0 / 27)$ and France $(2 / 68)$ [3,5]. However, the frequencies observed were lower than in other studies of ICU health care workers from other countries of the region such as Paraguay (20/142) and Colombia $(3 / 26)[6,7]$. The frequency of MRSA from this study was congruent with the average reported in the literature $(154 / 3309 ; 4.7 \%)$ for ICUs [1]. However, more samples are needed from health workers of other hospitals as well as from other areas in order to better estimate the prevalence of MRSA in ICUs. Studies from other hospital departments report a higher frequency than the present study.

A study of several hospital areas [3] reported an MRSA prevalence of 46/481 (9.6\%) in nursing staff. This prevalence rate was higher than the rates observed in the three hospital in the present study $(3 / 60,0 / 23,0 / 29)$. No MRSA was detected in medical or administration staff. The higher frequency of MRSA in nurses than in medical and administration staff is expected since nurses have contact with patients for prolonged periods of time. These results [8]are supported by other studies[8] where MRSA was not detected in medical staff; however, a compilation study [1] reported that on average, medical (79/991, $8 \%)$ and nursing $(186 / 2499,7.4 \%)$ staff had a similar prevalence of MRSA colonization.

MRSA colonization was also found in physiotherapy and cleaning and security staff (others) (Table 1). More data is needed to confirm if this group of health workers is susceptible to MRSA colonization. Even though a definitive conclusion annot be reached until more samples from these groups are analyzed, we would recommend that this set of workers be included in future epidemiology studies.

Studying the levels of MRSA colonization in health care workers is essential in order to prevent epidemic outbreaks of MRSA in hospitals. Transmission between patients and health care personnel is a very important source of infection. Albrich and Harbarth[ 1] found that 27 studies reported molecular and epidemiological information describing transmission of MRSA between patients and health care workers. The spread of MRSA from patients to health care workers (or vice versa) does not only occur within medical wards; it can also spread between different departments or hospitals. This is important because many health care workers are not confined to a single area and many of them have worked previously in other health care institutions. For instance, $70(33.3 \%)$ of participants from this study declared that they worked either currently or previously in other health care institutions over the past 15 years. Other factors such as age, male/female ratio, or length of service did not show significant differences (data not shown) and do not seem to be related to MRSA colonization in the present study.

Even though the objective of the present study was not to compare two MRSA detection methodologies, we observed that LightCycler MRSA advanced test was able to detect MRSA in five samples while CHROMagar detected MRSA in only three samples. LightCycler MRSA advanced test was significantly more sensitive than the culture method (CHROMagar MRSA) used in two previous studies $[9,10]$.

In summary, the frequency of MRSA colonization in health care personnel from the ICUs [1] of three hospital facilities is congruent with the average reported in the literature for ICUs. No significant differences in prevalence were observed between age, length of service, and job groups. MRSA was detected in nursing staff as well as physiotherapy staff and others. Medical and administration staff did not report MRSA colonization. Two limitations of this study were that both nares of each health care worker were not analyzed separately and that additional hospital areas (other than ICUs) were not sampled.

Even though colonization frequency was relatively low, hygienic measures and decolonization therapy for health care workers have both been reported to be effective measures of controlling epidemic outbreaks $[1,8]$. Regularly screening new patients or health care workers (especially if they rotate between areas) has been shown to have good results and to be a cost-effective measure [11,12]. Still, there is very limited information about the epidemiology of MRSA in Ecuadorian hospitals. Research on nosocomial infections in health care facilities could help to prevent epidemic outbreaks and improve health conditions of patients and health care personnel.

\section{Acknowledgements}

The authors wish to thank the ethical and scientific committees of the hospital involved in the study and Ing. Julio Sanchez for statistical advice. 


\section{References}

1. Albrich WC, Harbarth S (2008) Health-care workers: source, vector, or victim of MRSA? Lancet Infect Dis 8: 289-301.

2. Garrouste-Orgeas M, Timsit JF, Kallel H, Ben Ali A, Dumay MF, Paoli B, Misset B, Carlet J (2001) Colonization with methicillin-resistant Staphylococcus aureus in ICU patients: morbidity, mortality, and glycopeptide use. Inf Cont Hosp Epid 22: 687-692.

3. Eveillard M, Martin Y, Hidri N, Boussougant Y, Joly-Guillou ML (2004) Carriage of methicillin-resistant Staphylococcus aureus among hospital employees: prevalence, duration, and transmission to households. Inf Cont Hosp Epid 25: 114-120.

4. Shurland SM, Stine OC, Venezia RA, Johnson JK, Zhan M, Furuno JP, Miller RR, Johnson T, Roghmann MC (2009) Colonization sites of USA300 methicillin-resistant Staphylococcus aureus in residents of extended care facilities. Inf Cont Hosp Epid 30: 313-318.

5. Pan A, Lorenzotti S, Ferrari L, Granata L, Signorini L, Carnevale G (2006) Low rates of nasal colonization with methicillin-resistant Staphylococcus aureus among staff members of an Italian hospital. Inf Cont Hosp Epid 27: 218220.

6. Dávalos K, Báez S, Bianco H, Figueredo B, Carlos A, Ortellado J, Laconich M, Plans J, Ortiz C, Apodaca J, Paredes O (2008) Portación Nasal de Staphylococcus aureus en Personal Hospitalario en Unidades de Cuidados Intensivos Adultos. An Fac Cienc Méd (Asunción) XLI: 56-62.

7. Espinosa González CT, Romero Vanegas MK, Rincón Cruz G, Jácome Bohórquez M, Arámbula de Obregón AL (2011) Nasal carriers of Staphylococcus aureus in the personnel that works in a Hospital of Santander. Rev Univ Ind Santander, Salud 43: 111-117.

8. Scarnato F, Mallaret MR, Croizé J, Kouabenan DR, Dubois M, Maitre A, DeGaudemaris R (2003) Incidence and prevalence of methicillin-resistant Staphylococcus aureus nasal carriage among healthcare workers in geriatric departments: relevance to preventive measures. Inf Cont Hosp Epid 24: 456-458.

9. Peterson LR, Liesenfeld O, Woods CW, Allen SD, Pombo D, Patel PA, Mehta MS, Nicholson B, Fuller D, Onderdonk A (2010) Multicenter evaluation of the LightCycler methicillinresistant Staphylococcus aureus (MRSA) advanced test as a rapid method for detection of MRSA in nasal surveillance swabs. JClin Microbiol 48:1661-1666.

10. Danial J, Noel M, Templeton KE, Cameron F, Mathewson F, Smith M, Cepeda JA (2011) Real-time evaluation of an optimized real-time PCR assay versus Brilliance chromogenic MRSA agar for the detection of meticillin-resistant Staphylococcus aureus from clinical specimens. J of Med Microbiol 60: 323-328.

11. Lee BY, Bailey RR, Smith KJ, Muder RR, Strotmeyer ES, Lewis GJ, Ufberg PJ, Song Y, Harrison LH (2010) Universal methicillin-resistant Staphylococcus aureus (MRSA) surveillance for adults at hospital admission: an economic model and analysis. Inf Cont Hosp Epid 31: 598-606.

12. Blok HEM, Troelstra A, Kamp-Hopmans TEM, GigengackBaars ACM, Vandenbroucke-Grauls CMJE, Weersink AJL, Verhoef J, Mascini EM (2003) Role of healthcare workers in outbreaks of methicillin-resistant Staphylococcus aureus: a 10-year evaluation from a Dutch university hospital. Inf Cont Hosp Epid 24: 679-685.

\section{Corresponding author}

Jeannete Zurita

Servicio de Microbiología y Tuberculosis, Hospital Vozandes Villalengua Oe2-37 y Av. 10 de Agosto, Quito, Ecuador Phone: (593 2) 2262142

Fax: (593 2) 2269234

Email: jzurita@hcjb.org

Conflict of interests: No conflict of interests is declared. 